\title{
Asfixia por obstrucción de vía aérea con cuerpo extraño: hallazgos en autopsia médico legal.
}

\section{Asphyxia due to airway obstruction with foreign body: findings in a medico- legal autopsy.}

Karla Guerra ${ }^{1}$ ORCID ID: https://orcid.org/0000-0002-4661-1339, Arely Vásquez ${ }^{1}$, ORCID ID:https://orcid.org/0000-0001-9050-2458

${ }^{1}$ Departamento de Patología Forense, Dirección de Medicina Forense, Tegucigalpa.

Correspondencia a: heyarely76@gmail.com Recibido: 07 de febrero 2020 Aceptado: octubre 2020

Citar como: Guerra K, Vásquez A. Asfixia por obstrucción de vía aérea con cuerpo extraño: hallazgos en autopsia médico legal. Rev. Cienc. Forenses Honduras, 2020; 6(2): 31-32. DOl:https://doi.org $/ 10.5377 /$ rcfh.v6i2.10714

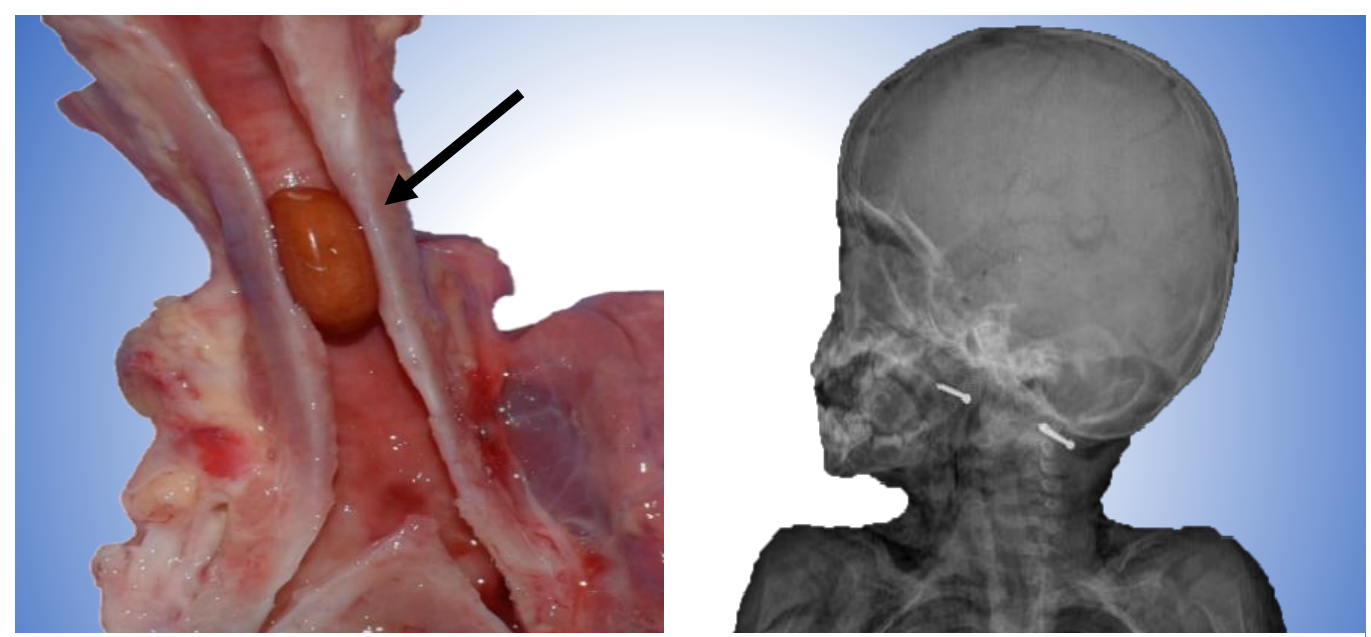

Figura 1A: Presencia de cuerpo extraño, una semilla de frijol crudo, obturando la tráquea (flecha). Figura 1B: Radiografía post mortem de barrido que no reveló la presencia del cuerpo extraño.

PALABRAS CLAVE: Autopsia, Cuerpo extraño, Vía aérea. KEY WORDS: Autopsy, Foreign body, Airway.

Femenina, de 14 meses de edad, sin antecedentes patológicos personales, que inició sintomatología de resfrió común, manejada en su hogar con remedios caseros, presentando súbitamente al séptimo día del inicio de los síntomas, dificultad respiratoria y cianosis generalizada. Fue llevada de emergencia a clínica periférica, donde al ser evaluada, presentó saturación de oxígeno en sangre de 45\%, por lo que fue referida a emergencia de pediatría de un hospital nacional donde se recibió en mal estado general, con enfisema subcutáneo y bradicardia; se intubó obteniendo contenido gástrico líquido, presentó falla multiorgánica, se realizaron maniobras de resucitación cardiopulmonar, no se logró estabilizar y falleció. Se remitió a autopsia médico legal, en la cual se encontró; obstrucción de vía aérea superior, congestión ocular, cianosis de labios y uñas, congestión visceral generalizada y de los huesos del cráneo. Alteraciones relevantes encontradas: 
presencia de cuerpo extraño (una semilla de frijol crudo) obturando la tráquea (Figura 1A). Después de la autopsia se concluyó que la causa de muerte era compatible con asfixia por obstrucción de vía aérea superior por cuerpo extraño y se determinó que la manera de la muerte fue accidental.

La aspiración de cuerpo extraño en la vía aérea se define como: "el ingreso de manera accidental o intencional a la laringe, tráquea o bronquios de un objeto, sea orgánico o inorgánico y que produce efectos en la respiración" $n^{1}$. Se presenta más frecuentemente en los niños menores de tres años y constituye una causa importante de mortalidad en menores entre 1 y 3 años ${ }^{2}$; su diagnóstico adecuado es fundamental ya que el retraso en el mismo puede ocasionar complicaciones y muerte. La historia clínica es de gran importancia, para el diagnóstico, ya que el examen físico y la radiografía de tórax pueden ser normales hasta en un tercio de los pacientes y usualmente estos no acuden inmediatamente a inspección médica; en un reporte de casos en Colombia se encontró que el $60 \%$ consultaron entre 3 y 10 días después del evento ${ }^{3}$, tal y como ocurrió en este caso en el cual la madre acudió a consulta hasta que los síntomas respiratorios de la menor eran graves. La radiografía post mortem de barrido no reveló la presencia del cuerpo extraño (Figura 1B), debido a que los cuerpos biológicos, como los vegetales no se visualizan en la radiografía, en este caso, en ella solo se evidenciaron los efectos secundarios a la obstrucción como: neumotórax, colapso pulmonar, discreta separación de la pleura entre otros.

Usualmente los niños con aspiración de cuerpo extraño presentan diversas manifestaciones clínicas, siendo las más comunes: tos, sibilancias y disminución de ruidos respiratorios. Se describen tres fases características en el cuadro clínico de la aspiración de cuerpo extraño. La primera ocurre en las 24 h siguientes a la aspiración y se caracteriza por un episodio agudo de atoramiento seguido de tos en el 91\% de los pacientes, náuseas y ocasionalmente dificultad respiratoria. La segunda fase es asintomática. En la tercera fase, el paciente sufre complicaciones asociadas a la presencia del cuerpo extraño en la vía aérea ${ }^{4}$. El diagnóstico temprano es fundamental, en este caso la madre no sospechó de un cuerpo extraño, por lo que no recurrió a evaluación médica temprana; asoció los síntomas a un resfriado común que trató con remedios naturales en su casa. La obstrucción por cuerpos extraños es frecuente en niños menores de tres años, siendo de suma importancia para el médico tratante sospechar en cuadros súbitos respiratorios la presencia de estos y evitar la muerte.

\section{REFERENCIAS BIBLIOGRÁFICAS}

1.-Yanowsky RG, Aguirre-Jáuregui OM, Rodríguez Franco E, Trujillo Ponce SA, Orozco Pérez J, Gutiérrez Padilla A, y col. Cuerpos extraños en vías aéreas. Arch Med [Internet]. 2013[citado 14 de mayo 2020]; 9(2):1-7 Disponible en: https://www.archivosdemedicina.com/medicina-de-familia/cuerpos-extraos-en-vasareas.pdf

2.-Bennicore Robledo A, Gutiérrez Morales G, Cuevas Schacht FJ. Aspiración de Cuerpo extraño. Acta Pediatr Mex [Internet]. 2019 [citado 14 de mayo 2020]; 40(3): 170-9. Disponible en: https://www.medigraphic.com/pdfs/actpedmex/apm-2019/apm193i.pdf

3. Correa C, González Casas DD, Rincón LC, Peña R, Luengas JP. Diagnóstico y tratamiento de cuerpos extraños en la vía aérea pediátrica: serie de casos. Pediatría. [Internet]. 2016 [citado 11 de marzo de 2020];49(4):1227. Disponible en: https://revistapediatria.org/rp/article/view/10

4.-Hegde SV, Hui PK, Lee EY. Tracheobronchial foreign bodies in children: imaging assessment. Semin Ultrasound CT MR. $2015 ; 36(1): 8-20$ 